Pacific Journal of Mathematics

HECKE EIGENFORMS AND REPRESENTATION NUMBERS O 


\title{
HECKE EIGENFORMS AND REPRESENTATION NUMBERS OF QUADRATIC FORMS
}

\author{
LYNNE H. WALLING
}

\begin{abstract}
Using the theory of modular forms and Hecke operators, we obtain arithmetic relations on average representation numbers of positive definite quadratic forms in an even number of variables.
\end{abstract}

1. Introduction. When looking for multiplicative relations satisfied by representation numbers of quadratic forms, it is natural to study the effect of the Hecke operators on theta series attached to quadratic forms; in this paper we use such theta series to construct Hecke eigenforms and thereby obtain relations on weighted averages of representation numbers of quadratic forms.

When working over the rationals we obtain the relations

$$
\begin{aligned}
\mathbf{r}\left(\operatorname{gen} L, m m^{\prime}\right)= & \lambda^{\prime}(m) \mathbf{r}\left(\operatorname{gen} L^{\prime}, m^{\prime}\right) \\
& -\sum_{\substack{a \mid\left(m, m^{\prime}\right) \\
a>1}} \chi_{L}(a) a^{k-1} \mathbf{r}\left(\operatorname{gen} L, \frac{m m^{\prime}}{a^{2}}\right)
\end{aligned}
$$

where $L$ is an even rank lattice equipped with a positive definite quadratic form, $\mathbf{r}($ gen $L, n)$ is the average number of times the lattices in the genus of $L$ represent $n, \lambda^{\prime}(m)$ is an easily computed constant, $\chi_{L}$ is a quadratic character associated to $L$, and $L^{\prime}$ is a particular sublattice of $L$ scaled by $1 / m$ (see Theorem 3.9).

We assume herein that we are working with a totally positive quadratic form $Q$ on vector space $\mathbf{V}$ of even dimension $2 k$ over a totally real number field $\mathbf{K}$; for a lattice $L$ on $\mathbf{V}$ we define the theta series attached to $L$ to be the Hilbert modular form

$$
\theta(L, \tau)=\sum_{x \in L} e^{\pi i \operatorname{Tr}(Q(x) \tau)} .
$$

We observe that there is a family of lattices related to $L$ which is partitioned into "nuclear families" such that the Hecke operators essentially permute the weighted averages of theta series attached to lattices within a nuclear family. Analyzing these permutations allows us to construct Hecke eigenforms, and analyzing the behavior of these 
eigenforms allows us to infer relations on the weighted averages of representation numbers of lattices within a nuclear family (as described above for $\mathbf{K}=\mathbf{Q}$ ).

Note that when the character associated to the lattice $L$ is nontrivial, the eigenforms we construct are only eigenforms for the subalgebra of the Hecke algebra which is known to map theta series to linear combinations of theta series with the same weight, level, and character.

2. Definitions. Let $\mathbf{K}$ be a totally real number field of degree $n$ over $\mathbf{Q}$; let $\mathscr{O}$ denotes its ring of integers and $\partial$ its different. Let $\mathscr{N}$ be an integral ideal, $\mathscr{I}$ a fractional ideal, $k \in \mathbf{Z}_{+}$, and $\chi_{\mathscr{N}}$ a numerical character modulo $\mathscr{N}$; we let

$$
\begin{array}{r}
\Gamma_{0}(\mathscr{N}, \mathscr{I})=\left\{\left(\begin{array}{ll}
a & b \\
c & d
\end{array}\right) \in\left(\begin{array}{cc}
\mathscr{O} & \mathscr{I}^{-1} \partial^{-1} \\
\mathscr{N} \mathscr{F} \partial & \mathscr{O}
\end{array}\right): a d-b c \in \mathscr{O}^{\times},\right. \\
a d-b c \gg 0\}
\end{array}
$$

and we let $\mathscr{M}_{k}\left(\Gamma_{0}(\mathscr{N}, \mathscr{I}), \chi_{\mathscr{N}}\right)$ denote the space of Hilbert modular forms of (uniform) weight $k$ and character $\chi_{\mathcal{N}}$ for the group $\Gamma_{0}(\mathscr{N}, \mathscr{I})$.

As defined in [8] (see also (2.21) of [4]), we have Hecke operators

$$
T(\mathscr{P}): \mathscr{M}_{k}\left(\Gamma_{0}(\mathscr{N}, \mathscr{I}), \chi_{\mathscr{N}}\right) \rightarrow \mathscr{M}_{k}\left(\Gamma_{0}(\mathscr{N}, \mathscr{\mathscr { P }}), \chi_{\mathscr{N}}\right)
$$

where $\mathscr{P}$ is a prime not dividing $2 \mathscr{N}$; we also have operators

$$
S(\mathscr{Q}): \mathscr{M}_{k}\left(\Gamma_{0}(\mathscr{N}, \mathscr{J}), \chi_{\mathscr{N}}\right) \rightarrow \mathscr{M}_{k}\left(\Gamma_{0}\left(\mathscr{N}, \mathscr{H}^{2}\right), \chi_{\mathscr{N}}\right)
$$

where $\mathscr{Q}$ is any fractional ideal relatively prime to $2 \mathscr{N}$ (i.e. $\operatorname{ord}_{\mathscr{P}} \mathscr{Q}$. $\operatorname{ord}_{\mathscr{P}} 2 \mathscr{N}=0$ for all finite primes $\mathscr{P}$ ). The collection of these operators $T(\mathscr{P})$ and $S(\mathscr{Q})$ generate a commutative algebra $\mathscr{T}$ which we call the Hecke algebra. Since the mapping from $\mathscr{M}_{k}\left(\Gamma_{0}(\mathscr{N}, \mathscr{I}), \chi_{\mathscr{N}}\right)$ onto $\mathscr{M}_{k}\left(\Gamma_{0}(\mathscr{N}, \alpha \mathscr{I}), \chi_{\mathscr{N}}\right)$ defined by $f \mapsto f \mid\left(\begin{array}{cc}\alpha^{-1} & 0 \\ 0 & 1\end{array}\right)$ (where $\alpha \gg 0$ ) is an isomorphism which commutes with the operators of $\mathscr{T}$, we set

$$
\mathscr{M}_{k}\left(\mathscr{N}, \chi_{\mathscr{N}}\right)=\bigoplus_{\mathscr{I}} \mathscr{M}_{k}\left(\Gamma_{0}(\mathscr{N}, \mathscr{I}), \chi_{\mathscr{N}}\right)
$$

where the sum runs over a complete set of strict ideal class representatives $\mathscr{I}$, and we consider $\mathscr{T}$ as an algebra of operators on $\mathscr{M}_{k}\left(\mathscr{N}, \chi_{\mathscr{N}}\right)$. (Notice that $f\left|\left(\begin{array}{ll}\alpha & 0 \\ 0 & 1\end{array}\right)=f\right|\left(\begin{array}{cc}\alpha^{\prime} & 0 \\ 0 & 1\end{array}\right)$ whenever $\alpha \gg 0$, $\alpha^{\prime} \gg 0$ and $\alpha \mathscr{I}=\alpha^{\prime} \mathscr{I}$.) We let $\mathscr{T}_{0}$ be the subalgebra of $\mathscr{T}$ which acts on each component space $\mathscr{M}_{k}\left(\mathscr{N}, \chi_{\mathscr{N}}\right)$; thus $\mathscr{T}_{0}$ is generated 
as a vector space by all operators of the form $T\left(\mathscr{M}_{1}\right) \cdots T\left(\mathscr{M}_{S}\right) S(\mathscr{J})$ where $\mathcal{J}$ is a fractional ideal relatively prime to $2 \mathscr{N}$, the $\mathscr{M}_{i}$ are integral ideals (not necessarily distinct) and $\alpha \mathscr{M}_{1} \cdots \mathscr{M}_{s} \mathscr{J}^{2}=\mathscr{O}$ for some $\alpha \gg 0$. As shown in Theorem 3.1 of [8], a $\mathscr{T}_{0}$-eigenform $f \in \mathscr{M}_{k}\left(\Gamma_{0}(\mathscr{N}, \mathscr{I}), \chi_{\mathscr{N}}\right)$ can be lifted to several linearly independent $\mathscr{T}$-eigenforms $F \in \mathscr{M}_{k}\left(\mathscr{N}, \chi_{\mathscr{N}}\right)$.

REMARK. The Hecke operators $T(\mathscr{P})$ defined here differ slightly from those defined in [2] and in [7]; letting $T^{\prime}(\mathscr{P})$ denote the latter operator, we have (up to identification of isomorphic spaces via the map $\left.f \mapsto f \mid\left(\begin{array}{ll}\alpha & 0 \\ 0 & 1\end{array}\right)\right)$

$$
N_{K / Q}(\mathscr{P})^{k / 2} T(\mathscr{P}) S\left(\mathscr{P}^{-1}\right)=T^{\prime}(\mathscr{P}) .
$$

We also have $S\left(\mathscr{P}^{-1}\right)=V(\mathscr{P})$ where $V(\mathscr{P})$ is the operator defined in [2] and [7].

Let $\mathbf{V}$ be a quadratic space of dimension $2 k$ over $\mathbf{K}$ with $Q$ a totally positive quadratic form and associated bilinear form $B$ such that $B(x, x)=Q(x)$. For $L$ a lattice on $\mathbf{V}$ we define the theta series attached to $L$ by

$$
\theta(L, \tau)=\sum_{x \in L} e^{\pi i \operatorname{Tr}(Q(x) \tau)}
$$

where $\tau \in \mathscr{H}^{n}$ (and $\mathscr{H}$ denotes the complex upper half-plane). As shown in [7] (see also [2]), $\theta(L, \tau)$ is a modular form of (uniform) weight $k$ with quadratic character $\chi_{L}$ for the group

$$
\Gamma_{0}^{1}(\mathscr{S}(L), \mathbf{n} L)=\left\{A \in \Gamma_{0}(\mathscr{S}(L), \mathbf{n} L): \operatorname{det} A=1\right\}
$$

where $\mathbf{n} L$, the norm of $L$, is the fractional ideal of $\mathscr{O}$ generated by $\frac{1}{2} Q(L)$, and $\mathscr{S}(L)$, the level or stufe of $L$, is the product of $(\mathbf{n} L)^{-1}\left(\mathbf{n} L^{\#}\right)^{-1}$ and perhaps some dyadic primes (see [7]). Here $L^{\#}$ denotes the dual of $L$; note that $\mathscr{S}(L)$ is an integral ideal and $\chi_{L}$ is a quadratic character modulo $\mathscr{S}(L)$. Also note that a nondyadic prime $\mathscr{P}$ divides $\mathscr{S}(L)$ if and only if $L_{\mathscr{P}}$ is not modular (as defined in [3]). (N.B.: the norm $\mathbf{n} L$ defined here is not the same as the norm defined in [3].)

Let $\mathscr{P}$ be a prime not dividing $2 \mathscr{S}(L)$; as shown in [7], when $L / \mathscr{P} L$ is hyperbolic we can realize $\theta(L, \tau) \mid T(\mathscr{P})$ as a linear combination of theta series attached to $\mathscr{P}$-sublattices of $L$, when $L / \mathscr{P} L$ is not hyperbolic we can realize $\theta(L, \tau) \mid T(\mathscr{P})^{2}$ as a linear combination of $\theta(\mathscr{P} L, \tau)$ and theta series attached to $\mathscr{P}^{2}$-sublattices of $L$, and $\theta(L, \tau) \mid S(\mathscr{P})$ is a constant multiple of $\theta(\mathscr{P} L, \tau)$. (A sublattice $L^{\prime}$ of $L$ is a $\mathscr{P}$-sublattice of $L$ if $\mathscr{P} L \subset L^{\prime} \subset L$ and 
$L^{\prime} / \mathscr{P} L$ is a maximal totally isotropic subspace of $L / \mathscr{P} L$; a $\mathscr{P}$-sublattice $L^{\prime \prime}$ of a $\mathscr{P}$-sublattice $L^{\prime}$ of $L$ is a $\mathscr{P}^{2}$-sublattice of $L$ if $\operatorname{dim} L^{\prime \prime} /\left(L^{\prime \prime} \cap \mathscr{P} L\right)=\operatorname{dim} L^{\prime} / \mathscr{P} L$.) Thus the subspace of $\mathscr{M}_{k}\left(\mathscr{S}(L), \chi_{L}\right)$ generated by forms whose components are theta series is invariant under the action of the subalgebra $\mathscr{T}^{L}$ of $\mathscr{T}$ where $\mathscr{T}^{L}$ is generated as an algebra by all operators $S(\mathscr{I}), T\left(\mathscr{P}^{2}\right)$, and by $T(\mathscr{P})$ when $L / \mathscr{P} L$ is hyperbolic. Thus the subspace of $\mathscr{M}_{k}\left(\Gamma_{0}(\mathscr{S}(L), \mathbf{n}(L)), \chi_{L}\right) \subseteq \mathscr{M}_{k}\left(\Gamma_{0}^{1}(\mathscr{S}(L), \mathbf{n}(L)), \chi_{L}\right)$ spanned by theta series is invariant under the subalgebra $\mathscr{T}_{0}^{L}=\mathscr{T}^{L} \cap \mathscr{T}_{0}$.

3. Constructing the eigenforms. From now on we set $\mathscr{N}=\mathscr{S}(L)$ and we require that for all dyadic primes $\mathscr{P}$, the localization $L_{\mathscr{P}}$ is an even unimodular lattice. As remarked in [8], $\chi_{L}=1$ if and only if $L / \mathscr{P} L$ is hyperbolic for all primes $\mathscr{P}+2 \mathscr{N}$. The space $L / \mathscr{P} L$ is hyperbolic if and only if $(-1)^{k} \operatorname{disc} L_{\mathscr{P}}=\pi^{2} \varepsilon$ where $\pi$ is some nonzero element of $\mathbf{K}_{\mathscr{P}}$ and $\varepsilon \in \mathscr{O}_{\mathscr{P}}^{\times}$such that $\varepsilon$ is a square modulo $\mathscr{P}$ (and disc $L_{\mathscr{P}}$ denotes the discriminant of $L_{\mathscr{P}}$ ); since $\operatorname{disc} L_{\mathscr{P}}=$ $\operatorname{disc} \mathbf{V}$ (in $\mathscr{O}_{\mathscr{P}}$ ) for all primes $\mathscr{P}$ and by $65: 19$ of [3] disc $\mathbf{V}$ is a square at an infinite number of primes, $L / \mathscr{P} L$ is hyperbolic for an infinite number of primes $\mathscr{P}$.

Definition. A lattice $K$ is in the family of $L$, denoted fam $L$, if $K$ is a lattice on $\mathbf{V}^{\alpha}$ for some $\alpha \gg 0, \alpha$ relatively prime to $2 \mathscr{N}$, and for every prime $\mathscr{P}$ there exist some $u_{\mathscr{P}} \in \mathscr{O}_{\mathscr{P}}^{\times}$such that $K_{\mathscr{P}}^{u_{\mathscr{P}}} \simeq L_{\mathscr{P}}$ (where $\simeq$ denotes isometry; here $\mathbf{V}^{\alpha}$ denotes the quadratic space $\mathbf{V}$ scaled by $\alpha$ and $K_{\mathscr{P}}^{u_{\mathscr{P}}}$ denotes the lattice $K_{\mathscr{P}}$ scaled by $\left.u_{\mathscr{P}}\right)$. We say $K \in \operatorname{fam} L$ is in the nuclear family of $L$, denoted $\operatorname{fam}^{+} L$, if there exists some $u \in \mathscr{O}^{\times}$such that $K^{u}$ is in the genus of $L$.

REMARK. In the case that $\chi_{L}=1$, the requirement that $\alpha$ be relatively prime to $2 \mathcal{N}$ is superfluous: For any $\beta \gg 0$, we can find some $\alpha \gg 0$ such that $\alpha$ is relatively prime to $2 \mathcal{N}$ and the Hilbert symbols $\left(\alpha,(-1)^{k} \operatorname{disc} V\right)_{\mathscr{P}}$ and $\left(\beta,(-1)^{k} \operatorname{disc} V\right)_{\mathscr{P}}$ are equal at all primes $\mathscr{P}$ dividing $2 \mathscr{N}$ (see $\S 63 \mathrm{~B}$ of [3]). (Note that if $\mathscr{P}$ is nondyadic then specifying the square class of $\beta$ in $\mathscr{O}_{\mathscr{P}}$ allows us to control $\left(\beta,(-1)^{k} \operatorname{disc} V\right)_{\mathscr{P}}$; if $\mathscr{P}$ is dyadic then, by the Local Square Theorem, taking $\beta \equiv \alpha(\bmod 4 \mathscr{P})$ we get $\beta=\alpha w^{2}$ for some $w \in$ $1+2 \mathscr{P}$ and hence $\left.\left(\beta,(-1)^{k} \operatorname{disc} V\right)_{\mathscr{P}}=\left(\alpha,(-1)^{k} \operatorname{disc} V\right)_{\mathscr{P}}.\right)$ For $\mathscr{P}$ a prime not dividing $2 \mathscr{N}$, $\operatorname{disc} V_{\mathscr{P}}=\operatorname{disc} L_{\mathscr{P}}$, and since $\chi_{L}=1, \S 3$ of [7] shows that $(-1)^{k} \operatorname{disc} L_{\mathscr{P}}$ is a square. Thus for every prime $\mathscr{P}$ we have $\left(\alpha,(-1)^{k} \operatorname{disc} V\right)_{\mathscr{P}}=\left(\beta,(-1)^{k} \operatorname{disc} V\right)_{\mathscr{P}}$, so by $66: 5$ of [3] we have $V^{\alpha} \simeq V^{\beta}$. 
It was remarked in [8] that the number of isometry classes in fam $L$ is finite when $\chi_{L}=1$; we now prove

LEMMA 3.1. The number of nuclear families in fam $L$ is $2^{r}$ where $r \in \mathbf{Z}$.

Proof. As shown in [7], $L_{\mathscr{P}}$ is modular at all primes $\mathscr{P}+2 \mathscr{N}$, so it follows from 92:1a of [3] that $L_{\mathscr{P}}^{u_{\mathscr{P}}} \simeq L_{\mathscr{P}}$ for all $u_{\mathscr{P}} \in \mathscr{O}_{\mathscr{P}}^{\times}$. In fact, for $\mathscr{P}+2$ and $u_{\mathscr{P}} \in \mathscr{O}_{\mathscr{P}}^{\times}$, we must have $L_{\mathscr{P}}^{u_{\mathscr{P}}} \simeq L_{\mathscr{P}}$ unless $L_{\mathscr{P}}$ has an odd rank Jordan component and $\left(u_{\mathscr{P}} \mid \mathscr{P}\right)=-1$ (see $92: 2$ of [3]). For $\mathscr{P} \mid 2$ and $u_{\mathscr{P}} \in \mathscr{O}_{\mathscr{P}}^{\times}$, the norm group of $L_{\mathscr{P}}$ is equal to the norm group of $L_{\mathscr{P}}^{u_{\mathscr{P}}}$ (since $L_{\mathscr{P}}$ is even unimodular), so by $93: 16$ of [3], $L_{\mathscr{P}}^{u_{\mathscr{P}}} \simeq L_{\mathscr{P}}$ if and only if $\mathbf{V}_{\mathscr{P}}^{u_{\mathscr{P}}} \simeq \mathbf{V}_{\mathscr{P}}$, and by $\S 63$ of [3], $\mathbf{v}_{\mathscr{P}}^{u_{\mathscr{P}}} \simeq \mathbf{V}_{\mathscr{P}}$ if and only if the Hilbert symbol $\left(u_{\mathscr{P}},(-1)^{k} \operatorname{disc} L_{\mathscr{P}}\right)_{\mathscr{P}}=\left(u_{\mathscr{P}},(-1)^{k} \operatorname{disc} \mathbf{V}_{\mathscr{P}}\right)_{\mathscr{P}}=1$.

Consider $\mathscr{P}$ to be a "bad" prime for $L$ if $\mathscr{P} \nmid 2$ and $L_{\mathscr{P}}$ has an odd rank Jordan component, or if $\mathscr{P} \mid 2$ and there exists some $u_{\mathscr{P}} \in$ $\mathscr{O}_{\mathscr{P}}^{\times}$such that $\left(u_{\mathscr{P}},(-1)^{k} \operatorname{disc} L_{\mathscr{P}}\right)_{\mathscr{P}}=-1$. Let $\mathscr{Q}_{1}, \ldots, \mathscr{Q}_{t}$ denote the "bad" primes for $L$. Thus there are at most $2^{t}$ genera in fam $L$. We associate to $K \in$ fam $L$ the vector $\left(\left(u_{1}: \mathscr{Q}_{1}\right), \ldots,\left(u_{t}: \mathscr{Q}_{t}\right)\right)$ where $K_{\mathscr{Q}_{1}}^{u_{t}} \simeq L_{\mathscr{Q}_{1}}$ and $(*: \mathscr{Q})$ is the Legendre symbol if $\mathscr{Q} \nmid 2$, and $(*: \mathscr{Q})$ is the Hilbert symbol $\left(*,(-1)^{k} \operatorname{disc} L_{\mathscr{Q}}\right)_{\mathscr{Q}}$ if $\mathscr{Q} \mid 2$; thus each genus within fam $L$ is associated to one such vector. We claim that these vectors associated to the genera in fam $L$ form a multiplicative subgroup of $\{ \pm 1\}^{t}$. Since gen $L$ is associated to $(1, \ldots, 1)$ and each vector has order 1 or 2 , we only need to verify that the set is closed. Take $J, K \in$ fam $L$. Thus $K$ is on $V^{\alpha}$ where $\alpha \gg 0, \alpha$ is relatively prime to $2 \mathscr{N}$, and gen $K$ is associated to the vector $\left(\left(\alpha: \mathscr{Q}_{1}\right), \ldots,\left(\alpha: \mathscr{Q}_{t}\right)\right)$. For any prime $\mathscr{P} \nmid 2 \mathscr{N}$, we have $K_{\mathscr{P}} \simeq K_{\mathscr{P}}^{u_{\mathscr{P}}} \simeq L_{\mathscr{P}}$, so $V_{\mathscr{P}}^{u_{\mathscr{D}}} \simeq V_{\mathscr{P}}$. From $\S 63$ of [3], we see this means

$$
1=\left(\alpha,(-1)^{k} \operatorname{disc} V\right)_{\mathscr{P}}=\left(\alpha,(-1)^{k} \operatorname{disc} L_{\mathscr{P}}\right)_{\mathscr{P}} .
$$

Since $L_{\mathscr{P}}$ is modular and of even rank, $\operatorname{disc} L_{\mathscr{P}}=\beta^{2} \varepsilon$ for some $\beta \in \mathbf{K}_{\mathscr{P}}$ and $\varepsilon \in \mathscr{O}_{\mathscr{P}}^{\times}$. If $\operatorname{ord}_{\mathscr{P}} \alpha$ is odd, then $(-1)^{k} \varepsilon$ must be a square, which implies that $L / \mathscr{P} L$ is hyperbolic (see $\S 3$ of [7]). Thus we have $\alpha \mathscr{P}_{1} \ldots \mathscr{P}_{s} \mathscr{I}^{2}=\mathscr{O}$ where the $\mathscr{P}_{i}$ are primes not dividing $2 \mathscr{N}$ such that $L / \mathscr{P} L$ (and hence $J / \mathscr{P} J$ ) is hyperbolic, and $\mathscr{I}$ is some fractional ideal. Let $J_{0}=J$, and let $J_{i}$ be a $\mathscr{P}_{i}$-sublattice of $J_{i-1}$. Then $\mathscr{I} J_{k}^{\alpha}$ must be in fam $L$, and for any "bad" prime $\mathscr{Q}$, $\left(\mathscr{I} J_{k}^{\alpha}\right)_{\mathscr{Q}}=J_{\mathscr{Q}}^{\alpha}$. Thus if gen $J$ is associated to the vector $\left(u_{1}, \ldots, u_{t}\right)$, 
the genus $\operatorname{gen}\left(\mathscr{I} J_{k}^{\alpha}\right)$ is associated to the vector $\left(u_{1}\left(\alpha: \mathscr{Q}_{1}\right), \ldots\right.$, $\left.u_{t}\left(\alpha: \mathscr{Q}_{t}\right)\right)$. Thus the set of vectors associated to the genera within fam $L$ forms a subgroup of the group $\{ \pm 1\}^{t}$.

A genus gen $K$ lies within $\operatorname{fam}^{+} L$ if and only if there is some totally positive unit $u$ such that gen $K^{u}=\operatorname{gen} L$; thus each nuclear family within fam $L$ contains the same number of genera, which is the number of distinct vectors in the set

$$
U_{L}=\left\{\left(\ldots,\left(u: \mathscr{Q}_{i}\right) \ldots\right): u \in \mathscr{O}^{\times}, u \gg 0\right\} .
$$

So each nuclear family is associated to a unique coset of $\{ \pm 1\}^{t} / U_{L}$, and thus there are $2^{r}$ nuclear families within fam $L$ where $r$ is some nonnegative integer.

REMARK. If $K$ is a $\mathscr{P}^{2}$-sublattice of $L$ then $\mathscr{P}^{-1} K \in \operatorname{gen} L \subseteq$ fam $^{+} L$ since at all primes $\mathscr{Q} \neq \mathscr{P}$ we have $K_{\mathscr{Q}}=L_{\mathscr{Q}}=\mathscr{P} L_{\mathscr{Q}}$, and $K_{\mathscr{P}} \simeq \mathscr{P} L_{\mathscr{P}}$ by construction. (For a more detailed discussion of $\mathscr{P}$ and $\mathscr{P}^{2}$-sublattices, see $\S 6$ of [7].)

Let $L_{1}, \ldots, L_{2^{r}}$ represent the distinct nuclear families within fam $L$, and define

$$
\theta\left(\mathrm{fam}^{+} L_{i}, \tau\right)=\sum_{K} \frac{1}{o(K)} \theta(K, \tau)
$$

where the sum is taken over a complete set of isometry class representations $K \in \mathrm{fam}^{+} L_{i}$ and $o(K)$. denotes the order of the orthogonal group $O(K)$ of $K$. Notice that

$$
\theta\left(\operatorname{fam}^{+} L_{i}, \tau\right) \in \mathscr{M}_{k}\left(\Gamma_{0}(\mathscr{N}, N(L)), \chi_{L}\right) .
$$

To help describe the action of $\mathscr{T}_{0}{ }^{L}$ on $\theta\left(\right.$ fam $\left.^{+} L_{i}, \tau\right)$, we first prove

LEMMA 3.2. Let $\mathscr{P}$ be a prime ideal such that $\mathscr{P}+2 \mathscr{N}$, and consider $T(\mathscr{P})$ as a map from $\mathscr{M}_{k}\left(\Gamma_{0}(\mathscr{N}, \mathbf{n} L), \chi_{L}\right)$ into $\mathscr{M}_{k}\left(\Gamma_{0}(\mathscr{N}, \mathscr{P} \mathbf{n} L), \chi_{L}\right)$.

(1) If $L / \mathscr{P} L$ is hyperbolic then

$$
\theta\left(\operatorname{fam}^{+} L, \tau\right) \mid T(\mathscr{P})=N_{K / Q}(\mathscr{P})^{k / 2}\left(N_{K / Q}(\mathscr{P})^{k-1}+1\right) \theta\left(\mathrm{fam}^{+} K, \tau\right)
$$

where $K$ is any $\mathscr{P}$-sublattice of $L$.

(2) If $L / \mathscr{P} L$ is not hyperbolic then

$$
\begin{aligned}
& \theta\left(\mathrm{fam}^{+} L, \tau\right) \mid T\left(\mathscr{P}^{2}\right) \\
& \quad=N_{K / Q}(\mathscr{P})^{k}\left(N_{K / Q}(\mathscr{P})^{2 k-2}-N_{K / Q}(\mathscr{P})^{k-1}+1\right) \theta\left(\mathrm{fam}^{+} \mathscr{P} L, \tau\right) .
\end{aligned}
$$


Proof. The first assertion follows from Lemma 5.2 of [8] and the observation that $\theta\left(\mathrm{fam}^{+} L, \tau\right)=\sum_{u} \theta\left(\operatorname{gen} L^{u}, \tau\right)$ where the sum runs over a finite set of totally positive units $u$ (which represent the distinct vectors in the set $U_{L}$ defined in the proof of the preceding lemma).

To prove the second assertion, we let $L_{1}^{\prime}, \ldots, L_{m}^{\prime}$ represent the distinct isometry classes in $\mathrm{fam}^{+} L$; as remarked above, any $\mathscr{P}^{2}$ sublattice $L^{\prime}$ of $L$ lies in fam $^{+} \mathscr{P} L$. We set $g_{i j}=$ the number of isometries $\sigma$ of $V$ which map $\mathscr{P} L_{j}^{\prime}$ to a $\mathscr{P}^{2}$-sublattice of $L_{i}^{\prime}$. Then we have that $\left(1 / o\left(L_{j}^{\prime}\right)\right) g_{i j}$ is the number of $\mathscr{P}^{2}$-sublattices of $L_{i}^{\prime}$ which are isometric to $\mathscr{P} L_{j}^{\prime},\left(1 / o\left(L_{i}^{\prime}\right)\right) g_{i j}$ is the number of $\mathscr{P}^{2}$. sublattices of $L_{j}^{\prime}$ which are isometric to $\mathscr{P} L_{i}^{\prime}$, and by Proposition 7.3 of [7],

$$
\begin{aligned}
& \sum_{j} \frac{1}{o\left(L_{j}^{\prime}\right)} g_{i j}=\sum_{i} \frac{1}{o\left(L_{i}^{\prime}\right)} g_{i j} \\
& \quad=N(\mathscr{P})^{k} N(\mathscr{P})^{k-2} \cdots N(\mathscr{P})^{0}\left(N(\mathscr{P})^{k}+1\right) \cdots\left(N(\mathscr{P})^{2}+1\right) .
\end{aligned}
$$

Now Proposition 6.1 and Theorem 7.4 of [7] yield the desired result.

Let gens $\mathscr{T}^{L}$ be the commutative monoid consisting of all (finite) products of the operators

$$
\begin{aligned}
\{T(\mathscr{P}) & : L / \mathscr{P} L \text { is hyperbolic }\} \\
& \cup\left\{T\left(\mathscr{P}^{2}\right): L / \mathscr{P} L \text { is not hyperbolic }\right\} \cup\{S(\mathscr{F})\}
\end{aligned}
$$

where it is understood that $\mathscr{P}$ is a prime ideal, $\mathscr{P}+2 \mathcal{N}$, and $\mathscr{I}$ is a fractional ideal relatively prime to $2 \mathscr{N}$. Then as vector spaces, $\mathscr{T}^{L}$ is generated by gens $\mathscr{T}^{L}$ and $\mathscr{T}_{0}{ }^{L}$ is generated by gens $\mathscr{T}_{0}{ }^{L}=$ $\mathscr{T}_{0} \cap$ gens $\mathscr{T}^{L}$. Now we prove

Lemma 3.3. Let $T \in$ gens $\mathscr{T}_{0}^{L}$. Then

$$
\theta\left(\text { fam }^{+} L_{i}, \tau\right) \mid T=\lambda_{T} \theta\left(\text { fam }^{+} L_{\sigma_{T}(i)}, \tau\right)
$$

where $\lambda_{T}$ is nonzero and dependent only on $T$, and $\sigma_{T}$ is a permutation of $\left\{1, \ldots, 2^{r}\right\}$. Furthermore, $\sigma_{T}^{2}=1$ and $\sigma_{T}$ has a fixed point only if $\sigma_{T}=1$; the set $\left\{\sigma_{T}: T \in\right.$ gens $\left.\mathscr{T}_{0}{ }^{L}\right\}$ forms a commutative group of order $2^{r}$ which acts transitively on $\left\{1, \ldots, 2^{r}\right\}$.

Proof. Let $\mathscr{Q}_{1}, \ldots, \mathscr{Q}_{t}$ be the "bad" primes for $L$ as in the proof of Lemma 3.1, and associate as before each genus within fam $L$ with a unique element of $\{ \pm 1\}^{t}$, and each nuclear family within fam $L$ with a unique element of the quotient group $\{ \pm 1\}^{t} / U_{L}$. 
Take $T \in$ gens $\mathscr{T}_{0}^{L}$. Thus

$$
T=T\left(\mathscr{P}_{1}\right) \cdots T\left(\mathscr{P}_{l}\right) T\left(\mathscr{P}_{l+1}^{2}\right) \cdots T\left(\mathscr{P}_{l+s}^{2}\right) S(\mathscr{I})
$$

where the $\mathscr{P}_{i}$ are primes (not necessarily distinct) such that $L / \mathscr{P}_{i} L$ is hyperbolic for $1 \leq i \leq l$ and not hyperbolic for $i>l$, and $\alpha \mathscr{P}_{1} \cdots \mathscr{P}_{l} \mathscr{P}_{l+1}^{2} \cdots \mathscr{P}_{l+s}^{2} \mathscr{I}^{2}=\mathscr{O}$ for some $\alpha \gg 0$. Let $K=K_{l+s} \subset$ $\cdots \subset K_{1} \subset K_{0}=L$ be lattices such that $K_{i}$ is a $\mathscr{P}_{i}$-sublattice of $K_{i-1}$ for $1 \leq i \leq l$, and $K_{i}$ is a $\mathscr{P}_{i}^{2}$-sublattice of $K_{i-1}$ for $i>l$. Then repeated use of the preceding lemma and Proposition 6.1 of [7] shows that $\theta\left(\right.$ fam $\left.^{+} L, \tau\right) \mid T=\lambda_{T} \theta\left(\right.$ fam $\left.^{+} \mathscr{I} K^{\alpha}, \tau\right)$ (where $\lambda \neq 0$ depends only on $T$ ); the techniques used in Lemma 3.1 show that fam $^{+} \mathscr{I} K^{\alpha}$ is associated to the coset $\left(\ldots,\left(\alpha \mid \mathscr{Q}_{i}\right), \ldots\right) U_{L}$. Similarly, if $\mathrm{fam}^{+} L_{i}$ is associated to $\left(\ldots, \varepsilon_{i}, \ldots\right) U_{L}$ then $\theta\left(\right.$ fam $\left.^{+} L_{i}, \tau\right) \mid T=$ $\lambda_{T} \theta\left(\operatorname{fam}^{+} L_{j}, \tau\right)$ where $\operatorname{fam}^{+} L_{j}$ is associated to $\left(\ldots, \varepsilon_{i}\left(\alpha \mid \mathscr{Q}_{i}\right), \ldots\right) U_{L}$. Hence we may associate to $T$ a permutation $\sigma_{T}$ of $\left\{1, \ldots, 2^{r}\right\}$ where $\sigma_{T}^{2}=1$ and $\sigma_{T}$ has a fixed point only when $\sigma_{T}=1$. Clearly $\left\{\sigma_{T}: T \in\right.$ gens $\left.\mathscr{T}_{0}^{L}\right\}$ is an abelian group of order $2^{r}$; that this group is transitive follows from Lemma 3.2 and the proof of Lemma 3.1.

This shows us that for $T \in$ gens $\mathscr{T}_{0}{ }^{L}$ we have

$$
\begin{aligned}
\sum a_{i} \theta\left(\mathrm{fam}^{+} L_{i}, \tau\right) \mid T & =\lambda_{T} \sum a_{i} \theta\left(\mathrm{fam}^{+} L_{\sigma_{T}(i)}, \tau\right) \\
& =\sum a_{\sigma_{T}(i)} \theta\left(\mathrm{fam}^{+} L_{i}, \tau\right)
\end{aligned}
$$

(since $\sigma_{T}^{2}=1$ ) so to find the eigenspaces of $\mathscr{T}_{0}^{L}$ on

$$
\bigoplus_{i}\left\langle\theta\left(\text { fam }^{+} L_{i}, \tau\right)\right\rangle
$$

we merely need to find the eigenspaces of $\left\{\sigma_{T}: \mathscr{T} \in\right.$ gens $\left.\mathscr{T}_{0}^{L}\right\}$ acting on $\mathbf{C}^{2^{r}}$ by $\left(a_{1}, a_{2}, \ldots\right) \mapsto\left(a_{\sigma_{T}(1)}, a_{\sigma_{T}(2)}, \ldots\right)$. Since $\sigma_{T}^{2}=1$ and $\left\{\sigma_{T}: \mathscr{T} \in\right.$ gens $\left.\mathscr{T}_{0}^{L}\right\}$ is transitive, a vector $\left(a_{1}, a_{2}, \ldots\right)$ is an eigenvector for $\left\{\sigma_{T}: \mathscr{T} \in\right.$ gens $\left.\mathscr{T}_{0}^{L}\right\}$ only if there is some $a \in \mathbf{C}$ such that $a_{i}= \pm a$ for each $i$. Clearly $(1,1, \ldots, 1)$ is an eigenvector; this corresponds to $\theta($ fam $L, \tau)=\sum_{i} \theta\left(\right.$ fam $\left.^{+} L_{i}, \tau\right)$, which was shown to be a $\mathscr{T}_{0}^{L}$-eigenform in [8] in the case that $\chi_{L}=1$.

LEMMA 3.4. For $1 \leq i \leq r$, let $\rho_{i 1}$ be the permutation

$$
\rho_{i 1}=\left(12^{i}\right)\left(22^{i}-1\right)\left(32^{i}-2\right) \cdots\left(2^{i-1} 2^{i-1}+1\right)
$$

and for $1<j \leq 2^{r-i}$, define $\rho_{i j}$ inductively by

$$
\rho_{i j}=\left(2^{i}+a_{1} 2^{i}+a_{2}\right) \cdots\left(2^{i}+a_{l-1} 2^{i}+a_{l}\right)
$$


where $\rho_{i j-1}=\left(\begin{array}{ll}a_{1} & a_{2}\end{array}\right) \cdots\left(a_{l-1} a_{l}\right)$. Set

$$
\sigma_{i}=\prod_{j=1}^{2^{r-i}} \rho_{i j}
$$

Then up to a reordering of $\operatorname{fam}^{+} L_{1}, \ldots$, fam $^{+} L_{2^{r}}$, the permutations $\sigma_{1}, \ldots, \sigma_{r}$ generate $\left\{\sigma_{T}: \mathscr{T} \in\right.$ gens $\left.\mathscr{T}_{0}^{L}\right\}$.

Proof. Clearly the $\sigma_{i}$ commute, $\sigma_{i}^{2}=1$, and for $i_{1}<i_{2}<\cdots<i_{l}$, we have

$$
\sigma_{i l} \cdots \sigma_{i_{2}} \sigma_{i_{1}}(1) \neq 1 \text {. }
$$

Hence if we demonstrate that $\sigma_{1}, \ldots, \sigma_{r} \in\left\{\sigma_{T}: T \in\right.$ gens $\left.\mathscr{T}_{0}^{L}\right\}$ then it must be the case that these $\sigma_{i}$ generate $\left\{\sigma_{T}: T \in\right.$ gens $\left.\mathscr{T}_{0}{ }^{L}\right\}$.

We begin by choosing $\sigma_{T_{1}} \neq 1$ and then ordering the nuclear families within fam $L$ such that $\sigma_{T_{1}}=\left(\begin{array}{ll}12 & 2\end{array}\right)(34) \cdots\left(2^{r}-12^{r}\right)=g_{1}$. Next, using the transitivity of the group $\left\{\sigma_{T}: T \in\right.$ gens $\left.\mathscr{T}_{0}{ }^{L}\right\}$, we choose $\sigma_{T_{2}}$ such that $\sigma_{T_{2}}(1)=4$. Now, $\sigma_{T_{1}} \sigma_{T_{2}}=\sigma_{T_{2}} \sigma_{T_{1}}$, so $\sigma_{T_{2}}(2)=3$. Thus $\sigma_{T_{2}}=\left(\begin{array}{ll}1 & 4\end{array}\right)\left(\begin{array}{ll}2 & 3\end{array}\right) *$. Next we observe that $\sigma_{T_{2}}(5) \neq 6$ else $\sigma_{T_{1}} \sigma_{T_{2}}$ is a nontrivial permutation with a fixed point; thus we can reorder the nuclear families such that $\sigma_{T_{2}}(5)=8$. Notice that we can choose this reordering to preserve the equality $\sigma_{T_{1}}=\sigma_{1}$. Since we have $\sigma_{T_{1}} \sigma_{T_{2}}=\sigma_{T_{2}} \sigma_{T_{1}}$, we get

$$
\sigma_{T_{2}}=\left(\begin{array}{ll}
1 & 4
\end{array}\right)\left(\begin{array}{ll}
2 & 3
\end{array}\right)\left(\begin{array}{ll}
5 & 8
\end{array}\right)\left(\begin{array}{ll}
6 & 7
\end{array}\right) * .
$$

Reasoning as above, $\sigma_{T_{2}}(9) \neq 10$, thus a reordering of $\left\{\mathrm{fam}^{+} L_{i}\right\}$ gives us

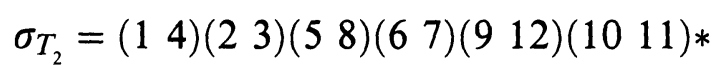

and we still have $\sigma_{T_{1}}=\sigma_{1}$. Continuing this process of reordering $\left\{\mathrm{fam}^{+} L_{i}\right\}$ gives us $\sigma_{T_{2}}=\sigma_{2}$ and $\sigma_{T_{1}}=\sigma_{1}$.

Now we choose $\sigma_{T_{3}}$ such that $\sigma_{T_{3}}(1)=8$; the subgroup $\left\langle\sigma_{T_{1}}, \sigma_{T_{2}}\right.$, $\left.\sigma_{T_{3}}\right\rangle$ is commutative and has no nontrivial elements with fixed points, so arguing as before we can reorder the nuclear families $\left\{\operatorname{fam}^{+} L_{i}\right\}$ so that $\sigma_{T_{3}}=\sigma_{3}, \sigma_{T_{2}}=\sigma_{2}$, and $\sigma_{T_{1}}=\sigma_{1}$. Continuing this process, we find $\sigma_{T_{4}}, \ldots, \sigma_{T_{r}} \in\left\{\sigma_{T}: T \in\right.$ gens $\left.\mathscr{T}_{0}{ }^{L}\right\}$ and a reordering of the nuclear families $\left\{\mathrm{fam}^{+} L_{i}\right\}$ such that $\sigma_{T_{r}}=\sigma_{r}, \ldots, \sigma_{T_{1}}=\sigma_{1}$.

This shows that the $\mathscr{T}_{0}^{L}$-eigenforms of the form $\sum_{i} a_{i} \theta\left(\mathrm{fam}^{+} L_{i}, \tau\right)$ correspond to the vectors $\left(\ldots, a_{i}, \ldots\right)$ which are eigenforms for 
$\left\{\sigma_{1}, \ldots, \sigma_{r}\right\}$. Now we show

LEMMA 3.5. Let $\sigma_{1}, \ldots, \sigma_{r}$ be as in Lemma 3.4. For $i=1, \ldots, r$, let $\mathbf{v}_{i}=\left(v_{i 1}, \ldots, v_{i 2^{r}}\right)$ where $v_{i j}=(-1)^{a}$ with $a \in \mathbf{Z}$ such that $a \cdot 2^{i-1}<j \leq(a+1) \cdot 2^{i-1}$. Then $\mathbf{v}_{1}, \ldots, \mathbf{v}_{r}$ generate an abelian group of order $2^{r}$ under componentwise multiplication, and every element in this group is an eigenvector for $\left\{\sigma_{T}: T \in\right.$ gens $\left.\mathscr{T}_{0}^{L}\right\}$ (where the permutation $\sigma$ maps the vector $\left(a_{1}, a_{2}, \ldots\right) \in\left(\mathbf{C}^{\times}\right)^{2^{r}}$ to $\left.\left(a_{\sigma(1)}, a_{\sigma(2)}, \ldots\right)\right)$. Furthermore, if $\mathbf{v}$ and $\mathbf{v}^{\prime}$ are distinct elements of this group, then $\mathbf{v}$ and $\mathbf{v}^{\prime}$ have distinct eigenvalues for some $\sigma_{T}$.

Proof. Notice that the entries of the vector $\mathbf{v}_{i}$ occur in blocks of $2^{i-1}$, so

$$
\sigma_{j}\left(\mathbf{v}_{i}\right)= \begin{cases}\mathbf{v}_{i} & \text { if } j<i, \\ -\mathbf{v}_{i} & \text { if } j \geq i .\end{cases}
$$

For $1 \leq j \leq r$ and $\mathbf{v}=\mathbf{v}_{i_{1}} \cdots \mathbf{v}_{i_{s}}$ with $i_{1}<\cdots<i_{s}$, take $l \leq s$ such that $j \geq i_{l}$ and $j<i_{l+1}$ if $l+1 \leq s$; then

$$
\sigma_{j}(\mathbf{v})= \begin{cases}\mathbf{v} & \text { if } l \text { is even }, \\ -\mathbf{v} & \text { if } l \text { is odd. }\end{cases}
$$

Since $\sigma\left(\mathbf{v} \mathbf{v}^{\prime}\right)=\sigma(\mathbf{v}) \sigma\left(\mathbf{v}^{\prime}\right)$, two vectors $\mathbf{v}$ and $\mathbf{v}^{\prime}$ have the same eigenvalues for all the $\sigma_{i}$ if and only if $\mathbf{v}=\mathbf{v}^{\prime}$.

REMARK. For any vectors $\mathbf{v}=\left(a_{1}, a_{2}, \ldots\right)$ in this group $\left\langle\mathbf{v}_{1}, \ldots\right.$, $\mathbf{v}_{r}$ ) we have $\sum_{i} a_{i}=0$ unless $\mathbf{v}=(1,1, \ldots, 1)$. To see this, write $\mathbf{v}=\mathbf{v}_{i_{1}} \cdots \mathbf{v}_{i_{s}}$ where $i_{1}<\cdots<i_{s}$, and observe that for $i<i_{s}$ and $j \equiv l\left(\bmod 2^{i_{s}-1}\right)$, we have

$$
v_{i j}= \begin{cases}v_{i l} & \text { if } i<i_{s} \text { or } j \equiv l\left(\bmod 2^{i-s}\right), \\ -v_{i l} & \text { if } i=i_{s} \text { and } j \not \equiv l\left(\bmod 2^{i}\right) .\end{cases}
$$

Thus $a_{j}=a_{l}$ if $j \equiv l\left(\bmod 2^{i_{s}}\right)$, and $a_{j}=-a_{l}$ if $j \equiv l\left(\bmod 2^{i_{s}-1}\right)$ but $j \not \equiv l\left(\bmod 2^{i_{s}}\right)$.

Let $\mathbf{v}_{r+1}, \ldots, \mathbf{v}_{2^{r}}$ denote the other vectors in the group generated by $\mathbf{v}_{1}, \ldots \mathbf{v}_{r}$, and write $\mathbf{v}_{i}=\left(v_{i 1}, \ldots, v_{i 2^{r}}\right)$. with the nuclear families of fam $L$ ordered as in Lemma 3.4, set

$$
E_{i}(\tau)=\sum_{j} v_{i j} \theta\left(\mathrm{fam}^{+} L_{j}, \tau\right)
$$

Then we have

THEOREM 3.6. Those $E_{i}(\tau)$ which are nonzero are linearly independent $T_{0}^{L}$-eigenforms. We also have

$$
\bigoplus_{i}\left\langle\theta\left(\mathrm{fam}^{+} L_{i}, \tau\right)\right\rangle=\bigoplus_{i}\left\langle E_{i}(\tau)\right\rangle
$$


so the number of forms $\theta\left(\mathrm{fam}^{+} L_{1}, \tau\right), \ldots, \theta\left(\mathrm{fam}^{+} L_{2^{r}}, \tau\right)$ which are linearly independent is equal to the number of $E_{i}(\tau)$ which are nonzero; here $2^{r}$ is the number of nuclear families within fam $L$.

Proof. Lemmas 3.3 and 3.5 show that for $T \in$ gens $\mathscr{T}_{0}{ }^{L}$,

$$
E_{i}(\tau) \mid T= \begin{cases}\lambda_{T} E_{i}(\tau) & \text { if } \sigma_{T}\left(\mathbf{v}_{i}\right)=\mathbf{v}_{i}, \\ -\lambda_{T} E_{i}(\tau) & \text { if } \sigma_{T}\left(\mathbf{v}_{i}\right)=-\mathbf{v}_{i}\end{cases}
$$

since $\mathbf{v}_{i}$ and $\mathbf{v}_{j}$ have the same eigenvalues for $\sigma_{1}, \ldots, \sigma_{r}$ only when $i=j$, the nonzero $E_{i}(\tau)$ must be linearly independent.

To finish proving the theorem, it suffices to show that the matrix $A=\left(v_{i j}\right)$ is nonsingular. Now, the $i, j$-entry of $A A^{t}$ is the $\operatorname{dot}$ product of $\mathbf{v}_{i}$ with $\mathbf{v}_{j}$, but this is just the sum of the entries of the vector $\mathbf{v}_{i} \mathbf{v}_{j}$. As remarked above, the sum of the entries of any vector $\mathbf{v}$ in the group generated by $\mathbf{v}_{1}, \ldots, \mathbf{v}_{r}$ is 0 if $\mathbf{v} \neq(1,1, \ldots, 1)$; thus $A A^{t}=2^{r} \cdot I$ where $I$ denotes the $2^{r} \times 2^{r}$ identity matrix.

Next we use Theorem 3.1 of [8] to lift the $\mathscr{T}_{0}^{L}$-eigenforms $E_{i}(\tau)$ to $\mathscr{T}^{L}$-eigenforms $\widetilde{E}_{i}(\tau) \in \mathscr{M}_{k}(\mathscr{N}, \chi)$ where $\chi$ is a Hecke character "extending $\chi_{L} \chi_{\infty}$ " with $\chi_{\infty}(\tilde{a})=\operatorname{sgn}\left(\tilde{a}_{\infty}\right)^{k}$ for an adele $\tilde{a} \in \mathbf{K}_{\mathbf{A}}$. (Thus $\chi$ is a Hecke character such that the finite part of its conductor divides the conductor of $\chi_{L}, \chi\left(\tilde{a}_{\mathscr{N}}\right)=\chi_{L}(a)$ when $\tilde{a}_{\mathscr{N}}$, the $\mathscr{N}$-part of the adele $\tilde{a}$, is a unit at all primes dividing $\mathscr{N}$ and $a \in \mathbf{K}^{\times}$such that $A \equiv \tilde{a}(\bmod \mathscr{N}) ; \mathrm{cf}$. [8] or [4]. Note that for $u \in \mathscr{O}^{\times}$,

$$
\begin{aligned}
& \chi_{L}(u) \theta\left(\operatorname{fam}^{+} L, \tau\right) \\
& \quad=\theta\left(\operatorname{fam}^{+} L, \tau\right) \mid\left(\begin{array}{cc}
u^{-1} & 0 \\
0 & u
\end{array}\right)=\operatorname{sgn}(u)^{k} \theta\left(\operatorname{fam}^{+} L, \tau\right)
\end{aligned}
$$

so $\chi_{L}(u)=\operatorname{sgn}(u)^{k}$ as required in [8].) The lift of $E_{i}(\tau)$ involves theta series attached to lattices in the "extended family" of $L$, which is defined as follows.

Definition. A lattice $K$ is connected to $L$ by a prime-sublattice chain if there exist lattices $K_{0}=L, K_{1}, \ldots, K_{s}$ such that $K_{i}$ is a $\mathscr{P}_{i}$ - or a $\mathscr{P}_{i}^{2}$-sublattice of $K_{i-1}$ (depending on whether $L / \mathscr{P}_{i} L$ is hyperbolic) and $K=\mathscr{I} K_{s}^{\alpha}$ for some fractional ideal $\mathscr{I}$ and some $\alpha \gg 0$. Here it is understood that $\mathscr{P}_{1}, \ldots, \mathscr{P}_{s}$ are prime ideals (not necessarily distinct) which do not divide $2 \mathscr{N}$, and $\mathscr{I}$ and $\alpha \mathscr{O}$ are relatively prime to $2 \mathscr{N}$; also, we take $K_{i}$ to be a $\mathscr{P}_{i}$-sublattice of $K_{i-1}$ if $L / \mathscr{P}_{i} L$ is hyperbolic, and we take $K_{i}$ to be a $\mathscr{P}_{i}^{2}$-sublattice of $K_{i-1}$ otherwise. If $K$ is connected by a prime-sublattice chain to 
a lattice in fam $L$ then we say $K$ is in the extended family of $L$, denoted $x$ fam $L$.

We can lift any $E_{i}(\tau)$ regardless of whether $E_{i}(\tau)$ is nonzero (although if $E_{i}(\tau)=0$ then the lift of $E_{i}(\tau)$ is also zero). Theorem 3.1 of [8] shows us that each nonzero $\mathscr{T}_{0}^{L}$-eigenform $E_{i}(\tau)$ can be lifted to $c_{i} h^{\prime}$ linearly independent $\mathscr{T}^{L}$-eigenforms $\widetilde{E}_{i}(\tau)$ where $h^{\prime}$ is the class number of $\mathbf{K} / \mathbf{Q}$ and $c_{i}$ is the cardinality of

$$
\left\{[\mathscr{Q}]: \mathscr{Q}=\alpha \mathscr{P}_{1} \cdots \mathscr{P}_{l} \mathscr{J}^{2} \text { with } \alpha \gg 0 \text { and } L / \mathscr{P}_{i} L \text { hyperbolic }\right\} ;
$$

here [Q] denotes the complex of the strict ideal class of $\mathscr{Q}$, and it is understood that $\mathscr{I}$ is a fractional ideal relatively prime to $2 \mathscr{N}$. Thus if $\chi_{L}=1$, each nonzero $E_{i}(\tau)$ can be lifted to $h$ linearly $\mathscr{T}$ eigenforms where $h$ is the strict class number of $\mathbf{K} / \mathbf{Q}$. Furthermore, an examination of this lift gives us

COROllaRY 3.7. For each $i=1, \ldots, 2^{r}$, each component of $\widetilde{E}_{i}(\tau)$ is a linear combination of theta series attached to lattices in $\operatorname{xfam} L$, and

$$
\widetilde{E}_{i}(\tau) \mid T(\mathscr{P})=\delta_{i}(\mathscr{P})\left(N_{K / Q}(\mathscr{P})^{k-1}+1\right) \widetilde{E}_{i}(\tau)
$$

where $\delta_{i}(\mathscr{P})= \pm 1$ so that $\sigma_{T(\mathscr{P})}\left(\mathbf{v}_{i}\right)=\delta_{i}(\mathscr{P}) \mathbf{v}_{i}$.

To obtain nice relations on the "Fourier coefficients" of the lifts $\widetilde{E}_{i}(\tau)$, we follow [4] and define the "Fourier coefficients" associated to an integral ideal $\mathscr{M}$ by

$$
\mathbf{a}_{i}(\mathscr{Q})=a_{\mu}(\xi) N_{K / Q}\left(\mathscr{J}_{\mu}\right)^{-k / 2}
$$

where $\xi \gg 0, \xi \mathscr{J}_{\mu}^{-1} i=\mathscr{M}$, and the form $\sum_{\zeta} a_{\mu}(\zeta) e^{2 \pi i \operatorname{Tr}(\zeta \tau)} \in$ $\mathscr{M}_{k}\left(\Gamma_{0}\left(\mathscr{N}, \mathscr{I}_{\mu}\right), \chi_{L}\right)$ is a component of $\widetilde{E}_{i}(\tau)$. Thus for $\xi \in \mathscr{N} L$, $\xi \gg 0$, and $\mathscr{M}=\xi(\mathbf{n} L)^{-1}$ we have

$$
\mathbf{a}_{i}(\mathscr{M})=N_{K / Q}(\mathbf{n} L)^{-k / 2} \sum_{j} v_{i j} \mathbf{r}\left(\operatorname{fam}^{+} L_{j}, \xi\right)
$$

where $\mathbf{r}\left(\right.$ fam $\left.^{+} L, \xi\right)=\sum_{L^{\prime}}\left(1 / o\left(L^{\prime}\right)\right) \mathbf{r}\left(L^{\prime}, \xi\right)$ with the sum running over isometry class representatives $L^{\prime} \in \operatorname{fam}^{+} L$, and where we take $\mathbf{n} L$ to be one of the strict ideal class representatives used to define $\mathscr{M}_{k}\left(\mathscr{N}, \chi_{L}\right)=\bigoplus_{\mathscr{I}} \mathscr{M}_{k}\left(\Gamma_{0}(\mathcal{N}, I), \chi_{L}\right)$. Since $\widetilde{E}_{i}(\tau)$ is an eigenform we have

$$
\kappa(\mathscr{M}) \mathbf{a}\left(\mathscr{M}^{\prime}\right)=\sum_{\mathscr{M}+\mathscr{M}^{\prime} \subseteq \mathscr{A}} \chi^{*}(\mathscr{A}) N_{K / Q}(\mathscr{A})^{k-1} \mathbf{a}_{i}\left(\mathscr{M} \mathscr{M}^{\prime} \mathscr{A}^{-2}\right)
$$


where $T(\mathscr{M}) \in \mathscr{T}^{L}$ and $\kappa(\mathscr{M})$ is some constant such that $\widetilde{E}_{i}(\tau) \mid T(\mathscr{M})$ $=\kappa(\mathscr{M}) \widetilde{E}_{i}(\tau)$ (see $(2.23)$ of [4]). These relations and a scrutiny of the lift will yield relations on the numbers $\mathbf{r}\left(\mathrm{fam}^{+} L, \xi\right)$; as a step toward that goal we prove

Lemma 3.8. For a prime $\mathscr{P} \nmid 2 \mathcal{N}$, define

$$
\varepsilon_{L}(\mathscr{P})= \begin{cases}1 & \text { if } L / \mathscr{P} L \text { is hyperbolic }, \\ -1 & \text { otherwise }\end{cases}
$$

and define

$$
\varepsilon_{L}(\mathscr{A})=\prod_{\mathscr{P} \mid \mathscr{A}} \varepsilon_{L}(\mathscr{P})^{\operatorname{ord}_{\mathscr{P}} \mathscr{A}}
$$

Then for any $a \in \mathscr{O}$ such that $a$ is relatively prime to $\mathscr{N}, \chi_{L}(a)=$ $\operatorname{sgn}(a)^{k} \varepsilon_{L}(a \mathscr{O})$. Moreover, if we let $\mathscr{I}_{1}, \ldots, \mathscr{I}_{h}$ be strict ideal class representatives such that

$$
\widetilde{E}_{i}(\tau) \in \mathscr{M}_{k}(\mathcal{N}, \chi) \subseteq \bigoplus_{\eta} \mathscr{M}_{k}\left(\Gamma_{0}\left(\mathscr{N}, \mathscr{I}_{\eta} \mathbf{n} L\right), \chi_{L}\right)
$$

(where $\chi$ is a Hecke character "extending $\chi_{L} \chi_{\infty}$ "- see the discussion following Lemma 3.6), then for $\mathscr{M}=\boldsymbol{\xi}(\mathbf{n} L)^{-1}$, with $\xi \in \mathbf{n} L, \xi \gg 0$, $\mathscr{M}$ relatively prime to $2 \mathscr{N}$ and $\mathscr{A}^{2} \mid \mathscr{M}$,

$$
\mathbf{a}_{i}\left(\mathscr{A}^{-2} \mathscr{M}\right)=\bar{\chi}^{*}(\mathscr{A}) \varepsilon_{L}(\mathscr{A}) N_{K / Q}(\mathbf{n} L)^{-k / 2} \cdot \sum_{j} v_{i j} \mathbf{r}\left(\operatorname{fam}^{+} \mathscr{A} L_{j}, 2 \xi\right)
$$

where $\chi^{*}(\mathscr{A})=\chi(\tilde{a})$ for an adele $\tilde{a}$ such that $\tilde{a}_{\mathscr{P}} \tilde{a}_{\infty}=1$ for all primes $\mathscr{P}$ with $\operatorname{ord}_{\mathscr{P}} \mathscr{A}=0$ and $\tilde{a} \mathscr{O}=\mathscr{A}$.

Proof. Letting $\left(\begin{array}{ll}a & b \\ c & d\end{array}\right) \in \Gamma_{0}(\mathcal{N}, \mathbf{n} L)$ such that $a d-b c=1$, we see that the restrictions on $d$ in the transformation formula (2) of [7] are unnecessary, but one needs to replace $N(d)^{-m / 2}$ by $d^{-m / 2}$. Then the arguments used to prove Theorem 3.7 of [7] show that $\chi_{L}(a)=$ $\operatorname{sgn}(a)^{k} \varepsilon_{L}(a)$ for any $a \in \mathscr{O}$ with $(a \mathscr{O}, \mathscr{N})=1$.

Now, take $\mu$ and $a \in \mathbf{K}^{\times}$such that $\mathscr{A}=a \mathscr{I}_{\mu}$ and $a \gg 0$. Then from the construction of $\widetilde{E}_{i}(\tau)$ we find that

$$
\begin{aligned}
\mathbf{a}_{i}\left(\mathscr{A}^{-2} \mathscr{M}\right)= & \bar{\chi}^{*}\left(\mathscr{J}_{\mu}\right) N_{K / Q}\left(\mathscr{I}_{\mu}\right)^{k} \\
& \cdot \sum_{j} v_{i j} \mathbf{r}\left(\operatorname{fam}^{+} \mathscr{I}_{\mu} L_{j}, 2 \alpha^{-2} \xi\right) \cdot N_{K / Q}\left(\mathbf{n}\left(\mathscr{I}_{\mu} L\right)\right)^{-k / 2} \\
= & \bar{\chi}^{*}\left(\mathscr{I}_{\mu}\right) \varepsilon_{L}\left(\mathscr{I}_{\mu}\right) N_{K / Q}(\mathbf{n} L)^{-k / 2} \sum_{j} v_{i j} \mathbf{r}\left(\mathrm{fam}^{+} \alpha \mathscr{I}_{\mu} L_{j}, 2 \xi\right) .
\end{aligned}
$$


Since $\chi_{L}$ is quadratic, we have

$$
\bar{\chi}^{*}(\mathscr{A}) \varepsilon_{L}(\mathscr{A})=\bar{\chi}^{*}\left(\mathscr{I}_{\mu}\right) \varepsilon_{L}\left(\mathscr{I}_{\mu}\right) \chi_{L}(a) \varepsilon_{L}(a \mathscr{O})=\bar{\chi}^{*}\left(\mathscr{I}_{\mu}\right) \varepsilon_{L}\left(\mathscr{I}_{\mu}\right),
$$

and so the lemma follows.

For a prime ideal $\mathscr{P} \nmid 2 \mathcal{N}$ we now define

$$
\lambda(\mathscr{P})=N_{K / Q}(\mathscr{P})^{k / 2}\left(N_{K / Q}(\mathscr{P})^{k-1}+1\right) \quad \text { when } \varepsilon_{L}(\mathscr{P})=1,
$$

and

$$
\begin{aligned}
\lambda\left(\mathscr{P}^{2}\right)= & N_{K / Q}(\mathscr{P})^{k}\left(N_{K / Q}(\mathscr{P})^{2 k-2}\right. \\
& \left.-N_{K / Q}(\mathscr{P})^{k-1}+1\right) \quad \text { when } \varepsilon_{L}(\mathscr{P})=-1 .
\end{aligned}
$$

We inductively define $\lambda(\mathscr{M})$ where $T(\mathscr{M}) \in \mathscr{T}^{L}$ by defining

$$
\lambda\left(\mathscr{P}^{a}\right) \lambda\left(\mathscr{P}^{b}\right)=\sum_{c=0}^{\min \{a, b\}} N_{K / Q}(\mathscr{P})^{c(2 k-1)} \lambda\left(\mathscr{P}^{a+b-2 c}\right)
$$

and $\lambda(\mathscr{M}) \lambda\left(\mathscr{M}^{\prime}\right)=\lambda\left(\mathscr{M} \mathscr{M}^{\prime}\right)$ whenever $\mathscr{M}$ and $\mathscr{M}^{\prime}$ are relatively prime.

THEOREM 3.9. Take $\xi \in \mathbf{n} L, \xi \gg 0$. Write $\xi(\mathbf{n} L)^{-1}=\mathscr{M}^{\prime}$ where and $\mathscr{M}^{\prime}$ are integral ideals and $T(\mathscr{M}) \in \mathscr{T}^{L}$. Then

$$
\begin{aligned}
\mathbf{r}\left(\mathrm{fam}^{+} L, 2 \xi\right)= & \lambda(\mathscr{M}) N_{K / Q}(\mathscr{M})^{-k / 2} \mathbf{r}\left(\mathrm{fam}^{+} L^{\prime}, 2 \xi\right) \\
& -\sum_{\substack{\mathscr{M}+\mathscr{M}^{\prime} \mathcal{A} \\
\mathscr{A} \neq \mathscr{\mathscr { C }}}} \varepsilon_{L}(\mathscr{A}) N_{K / Q}(\mathscr{A})^{k-1} \mathbf{r}\left(\mathrm{fam}^{+} \mathscr{A} L, 2 \xi\right)
\end{aligned}
$$

where $\mathbf{n} L^{\prime}=\mathscr{M} \mathbf{n} L$ and $L^{\prime}$ is connected to $L$ by a prime-sublattice chain. In the case that $\mathbf{K}=\mathbf{Q}$, we can scale $L$ to assume $\mathbf{n} L=\mathbf{Z}$; then for $m, m^{\prime} \in \mathbf{Z}$ such that $T(m) \in \mathscr{T}^{L}$ we have

$$
\begin{aligned}
\mathbf{r}\left(\operatorname{gen}^{+} L, 2 m m^{\prime}\right)= & \lambda(m) m^{-k / 2} \mathbf{r}\left(\operatorname{gen} L^{\prime}, 2 m^{\prime}\right) \\
& -\sum_{\substack{a \mid\left(m, m^{\prime}\right) \\
a>1}} \chi_{L}(a) a^{k-1} \mathbf{r}\left(\operatorname{gen} L, \frac{2 m m^{\prime}}{a^{2}}\right)
\end{aligned}
$$

where $L^{\prime} \in \operatorname{gen} L$ with gen $L^{\prime}$ determined by $m$.

Proof. Take $\xi \in \mathbf{n} L, \xi \gg 0$, and write $\boldsymbol{\xi}(\mathbf{n} L)^{-1}=\mathscr{M} \mathscr{M}^{\prime}$ with $T(\mathscr{M}) \in \mathscr{T}^{L}$ (i.e. $\mathscr{M}$ is relatively prime to $2 \mathscr{N}$ and $\operatorname{ord}_{\mathscr{P}} \mathscr{M}$ is even whenever $\varepsilon_{L}(\mathscr{P})=-1$ for $\left.\mathscr{P}+2 \mathscr{N}\right)$. To lift $E_{i}(\tau)$, we may as well assume that $\mathscr{M} \mathbf{n} L$ is one of the strict ideal class representatives used to define $\mathscr{M}_{k}(\mathscr{N}, \chi)$; then we observe that

$$
E_{i}(\tau) \mid T(\mathscr{M})^{2} S\left(\mathscr{M}^{-1}\right)=N_{K / Q}(\mathscr{M})^{-k} \lambda(\mathscr{M})^{2} E_{i}(\tau)
$$


so we can fix a square root of $\bar{\chi}^{*}(\mathscr{M})$ and define the $\mathscr{M} L$-component of $\widetilde{E}_{i}(\tau)$ to be

$$
\begin{aligned}
& \frac{\sqrt{\bar{\chi}^{*}(\mathscr{M})} N_{K / Q}(\mathscr{P})^{k / 2}}{\lambda(\mathscr{M})} E_{i} \mid T(\mathscr{M}) \\
& =\sqrt{\bar{\chi}^{*}(\mathscr{M})} N_{K / q}(\mathscr{M})^{k / 2} \sum_{j} v_{i j} \theta\left(L_{j}^{\prime}, \tau\right)
\end{aligned}
$$

where $\mathbf{n} L_{j}^{\prime}=\mathscr{M} \cdot \mathbf{n} L$ and $L_{j}^{\prime}$ is connected to $L_{j}$ by a prime-sublattice chain. (Notice that if $\mathscr{M}=\mathscr{J}^{2}$ then we can interpret

$$
\sqrt{\bar{\chi}^{*}(\mathscr{M})} N_{K / Q}(\mathscr{M})^{k / 2} \sum_{j} v_{i j} \theta\left(L_{j}^{\prime}, \tau\right)
$$

as $\varepsilon_{L}(\mathscr{I}) \bar{\chi}^{*}(\mathscr{I}) N_{K / Q}(\mathscr{I})^{k} \sum_{j} v_{i j} \theta\left(\mathscr{I} L_{j}, \tau\right)$ since $\mathscr{I} L_{j} \in \mathrm{fam}^{+} L_{j}^{\prime}$ in this case.) Then

$$
\widetilde{E}_{i}(\tau) \mid T(\mathscr{M})=\frac{\lambda(\mathscr{M})}{\sqrt{\bar{\chi}^{*}(\mathscr{M})} N_{K / Q}(\mathscr{M})^{k / 2}} \widetilde{E}_{i}(\tau) .
$$

Since $\mathscr{M}^{\prime}=\xi \mathscr{M}^{-1}(\mathbf{n} L)^{-1}$, we have

$$
\begin{aligned}
\mathbf{a}_{i}\left(\mathscr{M}^{\prime}\right)= & \sqrt{\bar{\chi}^{*}(\mathscr{M})} N_{K / Q}(\mathscr{M})^{k / 2} \sum_{j} v_{i j} \mathbf{r}\left(\operatorname{fam}^{+} L_{j}^{\prime}, 2 \xi\right) \\
& \cdot N_{K / Q}(\mathscr{M})^{-k / 2} N_{K / Q}(\mathbf{n} L)^{k / 2}
\end{aligned}
$$

so

$$
\begin{aligned}
& \kappa_{i}(\mathscr{M}) \mathbf{a}_{i}\left(\mathscr{M}^{\prime}\right) \\
& \quad=\lambda(\mathscr{M}) N_{K / Q}(\mathscr{M})^{-k / 2} N_{K / Q}(\mathbf{n} L)^{-k / 2} \sum_{j} v_{i j} \mathbf{r}\left(\operatorname{fam}^{+} L_{j}^{\prime}, 2 \xi\right)
\end{aligned}
$$

(where $\left.E_{i}(\tau) \mid T(\mathscr{M})=\kappa_{i}(\mathscr{M}) E_{i}(\tau)\right)$. Therefore we have

$$
\begin{aligned}
\mathbf{a}_{i}\left(\mathscr{M} \mathscr{M}^{\prime}\right)= & \kappa_{i}(\mathscr{M}) \mathbf{a}_{i}\left(\mathscr{M}^{\prime}\right) \\
& -\sum_{\substack{\mathscr{M}+\mathscr{M}^{\prime} \mathscr{C} \\
\mathscr{A} \neq \mathscr{Q}}} \chi^{*}(\mathscr{A}) N_{K / Q}(\mathscr{A})^{k-1} \mathbf{a}_{i}\left(\mathscr{M}^{\prime \prime} \mathscr{A}^{-2}\right)
\end{aligned}
$$

which, together with Corollary 3.7 and Lemma 3.8, give us

$$
\begin{aligned}
& \sum_{j} v_{i j} \mathbf{r}\left(\mathrm{fam}^{+} L_{j}, 2 \xi\right) \\
& =\lambda(\mathscr{M}) N_{K / Q}(\mathscr{M})^{-k / 2} \sum_{j} v_{i j} \mathbf{r}\left(\mathrm{fam}^{+} L_{j}^{\prime}, 2 \xi\right) \\
& -\sum_{\substack{\mathscr{M}+\mathscr{M}^{\prime} \mathscr{C} \\
\mathscr{A} \neq \mathscr{Q}}} \varepsilon_{L}(\mathscr{A}) N_{K / Q}(\mathscr{A})^{k-1} \sum_{j} v_{i j} \mathbf{r}\left(\mathrm{fam}^{+} \mathscr{A} L_{j}, 2 \xi\right) .
\end{aligned}
$$

Summing on $i$ and normalizing yields the desired result. 
4. Linear independence of theta series when $\mathbf{K}=\mathbf{Q}, \chi_{L}=1$, and $\operatorname{rank} L>2$. Suppose now that $\mathbf{K}=\mathbf{Q}, \chi_{L}=1$, and $\operatorname{rank} L=2 k$ with $k>1$. Since the object of this section is to show that the $E_{i}(\tau)$ constructed in $\S 3$ are nonzero (and thus by Theorem 3.6 the forms $\left\{\theta\left(\right.\right.$ fam $\left.\left.^{+} L_{i}, \tau\right)\right\}$ are linearly independent $)$, we may assume that the lattice $L$ has been scaled so that $\mathbf{n} L=\mathbf{Z}$. Let $q_{1}, \ldots, q_{t}$ denote the "bad" primes for $L$ (as defined in the proof of Lemma 3.1). Since $L$ is even unimodular when localized at 2 , an analysis of the Hilbert symbol $\left(*,(-1)^{k} \operatorname{disc} L\right)_{2}$ shows that 2 is not a "bad" prime for $L$. Furthermore, since $\chi_{L}=1$, there are $2^{t}$ nuclear families within fam $L$. (Using the Chinese Remainder Theorem we can realize each vector in $\{ \pm 1\}^{t}$ as $\left(\left(a: q_{1}\right), \ldots,\left(a: q_{t}\right)\right)$ where $a=p_{1} \cdots p_{s}$ with the $p_{i}$ primes (not necessarily distinct) and $p_{i}+2 \mathscr{N}$. Since $L / p_{i} L$ is hyperbolic, $K^{1 / a} \in$ fam $L$ where $K=K_{s}, K_{0}=L$, and $K_{i}$ is a $p_{i^{-}}$ sublattice of $K_{i-1}$. Then fam $^{+} K^{1 / a}=\operatorname{gen} K^{1 / a}$ is associated to the vector $\left(\left(a: q_{1}\right), \ldots,\left(a: q_{t}\right)\right)$.) For any prime $p$ the localized lattice $L_{p}$ has the Jordan decomposition

$$
L_{p} \simeq J_{1} \perp \cdots \perp J_{l}
$$

letting $e_{s}=\operatorname{ord}_{p} \mathbf{n} J_{s}$ and $m_{s}=\operatorname{rank} J_{s}=\operatorname{dim} J_{s} \otimes \mathbf{Q}$, for $b>0$ with $\operatorname{ord}_{p} b=e$ we define

$$
\operatorname{rank}_{p}(b, L)=\prod_{e_{s}<e}\left(e-e_{s}\right) m_{s} .
$$

Notice that $\operatorname{rank}_{p}(b, L)$ depends on $\operatorname{fam} L, p$ and $b$; since fam $L$ is fixed, we simply write $\operatorname{rank}_{p}(b)$ for $\operatorname{rank}_{p}(b, L)$.

LEMMA 4.1. Fix a prime $q$ and suppose $L^{\prime} \in$ fam $L$ such that for all primes $p$ we have $L_{p}^{\prime} \simeq L_{p}$ except when $p=q$. (Thus $q$ must be a "bad" prime for $L$.) Then

$$
\begin{aligned}
& \theta\left(\mathrm{fam}^{+} L, \tau\right)-\theta\left(\mathrm{fam}^{+} L^{\prime}, \tau\right) \\
& =2 \operatorname{mass} L \cdot \sum_{\substack{b>0,(a, b)=1 \\
\operatorname{rank}_{q} b \text { odd }}} H\left(\frac{a}{b}, L\right)(b \tau-a)^{-k}
\end{aligned}
$$

and

$$
\begin{aligned}
& \theta\left(\mathrm{fam}^{+} L, \tau\right)+\theta\left(\mathrm{fam}^{+} L^{\prime}, \tau\right) \\
& \quad=2 \operatorname{mass} L \cdot\left[1+\sum_{\substack{b>0,(a, b)=1 \\
\operatorname{rank}_{q} b \text { even }}} H\left(\frac{a}{b}, L\right)(b \tau-a)^{-k}\right]
\end{aligned}
$$


where

$$
H\left(\frac{a}{b}, L\right)=i^{k}(\operatorname{disc} L)^{-1 / 2} b^{-k} \sum_{x \in L / b L} e^{\pi i(a / b) Q(x)}
$$

and mass $L=\sum_{L^{\prime} \in \mathrm{fam}^{+} L} \frac{1}{o\left(L^{\prime}\right)}$ is an invariant for fam $L$.

Proof. First we verify that mass $L$ is an invariant of fam $L$. From our construction we know that the zero-Fourier coefficient of $\theta\left(\right.$ fam $\left.^{+} L, \tau\right)$ is mass $L$; from the definition of Hecke operators we know that the zero-Fourier coefficient of $\theta\left(\mathrm{fam}^{+} L, \tau\right) \mid T(p)$ must be $\left(p^{k-1}+1\right)$ times the zero-Fourier coefficient of $\theta\left(\right.$ fam $\left.^{+} L, \tau\right)$. Using Lemma 3.2 and identifying isomorphic spaces of modular forms via the map

$$
f \mapsto f \mid\left(\begin{array}{ll}
1 & 0 \\
0 & p
\end{array}\right),
$$

we see that for $p$ a prime such that $\chi_{L}(p)=1$ we have

$$
\theta\left(\operatorname{fam}^{+} L, \tau\right) \mid T(p)=\left(p^{k-1}+1\right) \theta\left(\mathrm{fam}^{+} K^{1 / p}, \tau\right)
$$

where $K$ is a $p$-sublattice of $L$ and hence $K^{1 / p} \in$ fam $L$. Since the zero-Fourier coefficient of $\theta\left(\mathrm{fam}^{+} K^{1 / p}, \tau\right)$ is mass $K^{1 / p}$, we have that mass $L=\operatorname{mass} K^{1 / p}$. Now, as discussed at the beginning of this section, any nuclear family $\operatorname{fam}^{+} L^{\prime} \subseteq$ fam $L$ has a representative of the form $\left(K_{s}\right)^{1 / a}$ where $a=p_{1} \cdots p_{s}$ with $p_{1}, \ldots, p_{s}$ primes (not necessarily distinct) such that $\chi_{L}\left(p_{i}\right)=1$ and $K_{0}=L, K_{1}, \ldots, K_{s}$ are lattices with $K_{i}$ a $p_{i}$-sublattice of $K_{i-1}$; thus it follows that mass $L^{\prime}=$ mass $L$ for any $L^{\prime} \in$ fam $L$.

From equation (82) of [5] we have

$$
\theta\left(\mathrm{fam}^{+} L, \tau\right)=\operatorname{mass} L\left[1+\sum_{a, b} H\left(\frac{a}{b}, L\right)(b \tau-a)^{-k}\right]
$$

where the sum is over all integers $a$ and $b$ with $b>0$ and $(a, b)=1$, and

$$
H\left(\frac{a}{b}, L\right)=i^{k}(\operatorname{disc} L)^{-1 / 2} b^{-k} \sum_{x \in L / b L} \mathrm{e}\left(\frac{a}{b} Q(x)\right)
$$

(here we write $\mathrm{e}(\alpha)$ for $\left.\mathrm{e}^{\pi i \alpha}\right)$. Using the results of $\S 3$ of [7] we have

$$
\sum_{x \in L / b L} \mathrm{e}\left(\frac{a}{b} Q(x)\right)=\left(b^{\prime \prime}\right)^{k} \chi_{L}\left(b^{\prime \prime}\right) \cdot \prod_{\substack{p \text { prime } \\ p^{c} \| b^{\prime}}}\left(\sum_{\substack{x \in p^{-e} b L / b L \\ \mathrm{e}}} \mathrm{e}\left(\frac{a}{b} Q(x)\right)\right)
$$

where $b=b^{\prime} b^{\prime \prime}$ with $\left(b^{\prime}, b^{\prime \prime}\right)=1=\left(b^{\prime \prime}, N\right)$. Fix a prime $p \mid b^{\prime}$ and let 
$e=\operatorname{ord}_{p} b$; by assumption $p \neq 2$ (since $L$ is even and unimodular when localized at 2 and so $2 \nmid N$; see $\S 3$ of [7]) and hence we can write

$$
L_{p} \simeq J_{1} \perp \cdots \perp J_{l}
$$

where $\operatorname{rank} J_{s}=m_{s}$ and $J_{s} \simeq p^{e_{s}}\left\langle 1, \ldots, 1, \varepsilon_{s}\right\rangle$ with $\varepsilon_{s} \in \mathbf{Z}_{p}^{\times}$and $e_{s}<e_{s+1}$. Since we are assuming $\mathbf{Z}=\mathbf{n} L$ we have $e_{1}=0$. We know $L / p^{e} L \approx L_{p} / p^{e} L_{p}$ (where the isomorphism is as $\mathbf{Z} / p^{e} \mathbf{Z}$-modules), and if $x \in L$ and $x^{\prime} \in L_{p}$ such that $x-x^{\prime} \in p^{e} L_{p}$ then

$$
Q(x) \equiv Q\left(x^{\prime}\right) \quad\left(\bmod p^{e} \mathbf{Z}\right) \text {. }
$$

Thus, choosing $\mu \in 2 \mathbf{Z}$ such that $\mu \equiv p^{e} / b\left(\bmod p^{e}\right)$, the techniques of $\S 3$ of [7] give us

$$
\begin{aligned}
& \sum_{x \in p^{-e} b L / b L} \mathrm{e}\left(\frac{a}{b} Q(x)\right) \\
& =\prod_{s=1}^{l}\left[\sum_{d_{1}, \ldots, d_{m_{s}} \in \mathbf{Z} / p^{e} \mathbf{Z}} \mathrm{e}\left(\mu a p^{e_{s}-e}\left(d_{1}^{2}+\cdots+d_{m_{s}-1}^{2}+\varepsilon_{s} d_{m_{s}}^{2}\right)\right)\right] \\
& =p^{2 k e} \prod_{e_{s}<e} p^{m_{s}\left(e_{s}-e\right) / 2} c_{s}\left(\frac{a}{b}, L, p\right)
\end{aligned}
$$

where

$$
\begin{aligned}
c_{s}\left(\frac{a}{b}, L, p\right) \\
\quad=\left\{\begin{array}{l}
1 \quad \text { if } 2 \mid\left(e-e_{s}\right), \\
\left((-1)^{m-s / 2} \mid p\right) \text { if } 2 \nmid\left(e-e_{s}\right) \text { but } 2 \mid m_{s}, \\
\left((-1)^{m_{s} / 2-1} a \varepsilon_{s} \mid p\right) p^{-1 / 2} \sum_{d \in \mathbf{Z} / p \mathbf{Z}} \mathrm{e}\left(\mu d^{2}\right)
\end{array} \text { if } 2 \nmid\left(e-e_{s}\right) m_{s} .\right.
\end{aligned}
$$

(Recall that $e_{1}=0$ since $\mathbf{n} L=\mathbf{Z}$, so $e>e_{1}$.) For $L^{\prime} \in$ fam $L$ such that $L_{p}^{\prime} \simeq L_{p}^{u}$ with $u \in \mathbf{Z}_{p}^{\times}$, we see that $c_{s}\left(\frac{a}{b}, L^{\prime}, p\right)=(u \mid p) c_{s}\left(\frac{a}{b}, L, p\right)$ if $e_{s}<e$ and $2 \nmid\left(e_{s}\right) m_{s}$, and $c_{s}\left(\frac{a}{b}, L^{\prime}, p\right)=c_{s}\left(\frac{a}{b}, L, p\right)$ otherwise. Since $(u \mid p)=-1$ only when $p=q$, the lemma now follows.

Now we can prove

LeMma 4.2. For each $i=1, \ldots, 2^{t}$ we have

$$
E_{l}(\tau)=\operatorname{mass} L \cdot\left[\prod_{j=1}^{t}\left(1+(-1)^{\delta_{\imath \jmath}}\right)+2^{t} \sum_{a, b} H\left(\frac{a}{b}, L\right)(b \tau-a)^{-k}\right]
$$

where the sum runs over all integers $a, b$ with $b>0,(a, b)=1$, 
and for each $j, \delta_{i j}$ is chosen to be 0 or 1 such that $\operatorname{rank}_{q_{j}} b \equiv \delta_{i j}$ $(\bmod 2)$. Also, $\prod_{j=1}^{t}\left(1+(-1)^{\delta_{i j}}\right)=0$ unless $E_{i}(\tau)=\theta(\operatorname{fam} L, \tau)$.

Proof. Choose $T_{1}, \ldots, T_{t} \in$ gens $\mathscr{T}^{L}$ such that $\theta\left(\mathrm{fam}^{+} L, \tau\right) \mid T_{j}=$ $\lambda_{T_{1}} \theta\left(\right.$ fam $\left.^{+} L^{\prime}, \tau\right)$ for some $L^{\prime} \in$ fam $L$ with $L_{q_{s}} \simeq L_{q_{s}}^{\prime}$ except when $s=j$. Then we can arrange the nuclear families within fam $L$ such that $\sigma_{j}=\sigma_{T}, 1 \leq j \leq t$, where $\sigma_{j}$ is as defined in Lemma 3.4. Let

$$
\delta_{i j}= \begin{cases}0 & \text { if } \sigma_{j}\left(\mathbf{v}_{i}\right)=\mathbf{v}_{i}, \\ 1 & \text { if } \sigma_{j}\left(\mathbf{v}_{i}\right)=-\mathbf{v}_{i}\end{cases}
$$

(where $\mathbf{v}_{i}$ is defined in Lemma 3.5 and $E_{i}(\tau)=\sum_{j=1}^{t} v_{i j} \theta\left(\right.$ fam $\left.^{+} L_{j}, \tau\right)$ ). Then the argument used to prove Lemma 4.1 now gives us $\frac{1}{\operatorname{mass} L} E_{i}(\tau)$

$$
\begin{aligned}
& =\left(1+(-1)^{\delta_{u l}}\right)+2 \sum_{j=1}^{2^{t-1}}\left(\sum_{\operatorname{rank}_{q_{1}} \equiv \delta_{l 1}(2)} H\left(\frac{a}{b}, L_{j}\right)(b \tau-a)^{-k}\right) \\
& =\left(1+(-1)^{\delta_{u t}}\right)\left(1+(-1)^{\delta_{i t-1}}\right) \\
& +4 \sum_{j=1}^{2^{t-2}}\left(\sum_{\substack{\operatorname{rank}_{q_{1}} b \equiv \delta_{i 1}(2) \\
\operatorname{rank}_{q_{2}} b \delta_{i 2}(2)}} H\left(\frac{a}{b}, L_{j}\right)(b \tau-a)^{-k}\right)
\end{aligned}
$$

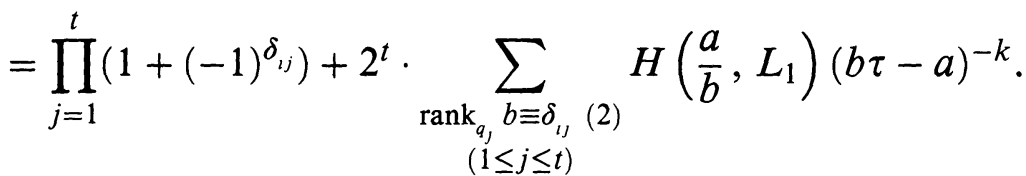

Finally we have

THEOREM 4.3. For each $i=1, \ldots, 2^{t}$, the form $E_{i}(\tau)$ is nonzero, and hence the set $\left\{\theta\left(\mathrm{fam}^{+} L_{1}, \tau\right), \ldots, \theta\left(\mathrm{fam}^{+} L_{2^{t}}, \tau\right)\right\}$ is linearly independent.

Proof. If $E_{i}(\tau)=\theta($ fam $L, \tau)$ then the zero-Fourier coefficient of $E_{i}(\tau)$ is nonzero. So suppose $E_{i}(\tau) \neq \theta(\operatorname{fam} L, \tau)$; we will show that the first Fourier coefficient of $E_{i}(\tau), a_{i}(1)$, is nonzero. As before, let $q_{1}, \ldots, q_{t}$ denote the "bad" primes for $L$ and set $Q=q_{1}^{\delta_{11}} \cdots q_{t}^{\delta_{t}}$ where the $\delta_{i j}$ are as defined in the preceding proof. From the 
preceding lemma we have

$$
\begin{aligned}
\left(2^{t} \text { mass } L\right)^{-1} E_{i}(\tau) & \left(\sum_{\substack{a \in \mathbf{Z} \\
(a, b)=1}} H\left(\frac{a}{b}, L\right)(b \tau-a)^{-k}\right) \\
= & \sum_{\substack{\operatorname{rank}_{q_{i}} b \delta_{l j}(2) \\
=}} H\left(\frac{1}{b}, L\right) \sum_{a \in(\mathbf{Z} / b \mathbf{Z})^{\times}}(a \mid Q) \sum_{m \in \mathbf{Z}}(b \tau-a+b m)^{-k} \\
= & \sum_{b} H\left(\frac{1}{b}, L\right) \sum_{a \in(\mathbf{Z} / b \mathbf{Z})^{\times}}(a \mid Q) \frac{(-1)^{k-1} 2 k \zeta(k)}{B_{k}} \times \sum_{m=1}^{\infty} m^{k-1} \mathrm{e}^{2 \pi i m(\tau-a / b)} \\
= & \frac{(-1)^{k-1} 2 k \zeta(k)}{B_{k}} \sum_{b} b^{-k} H\left(\frac{1}{b}, L\right) \\
& \sum_{a \in(\mathbf{Z} / b \mathbf{Z})^{\times}}\left(\sum_{m=1}^{\infty}(-a \mid Q) m^{k-1} \mathrm{e}^{2 \pi i m a / b} \mathrm{e}^{2 \pi i m \tau}\right) .
\end{aligned}
$$

(Here $\zeta$ denotes the Riemann-zeta function, and $B_{k}$ is the $k$ th Bernoulli number.) Now, take $\widetilde{Q} \in \mathbf{Z}$ such that

$$
\operatorname{ord}_{p} \widetilde{Q}= \begin{cases}0 & \text { if } p \nmid b, \\ 1 & \text { if } \operatorname{ord}_{p} Q=1, \\ 2 & \text { otherwise }\end{cases}
$$

then for $b \geq 1$ with $\operatorname{rank}_{q_{l}} b \equiv \delta_{i j}(\bmod 2)$, we have

$$
\begin{aligned}
& \sum(-a \mid Q) \mathrm{e}^{2 \pi i m a / b} \\
& a \in(\mathbf{Z} / b \mathbf{Z})^{\times} \\
& =\sum_{a \in \mathbf{Z} / b \mathbf{Z}}(-a \mid \widetilde{Q}) \mathrm{e}^{2 \pi i m a / b} \\
& =\prod_{q^{e} \| b}\left(\frac{-b}{q^{e}} \mid q\right)^{\operatorname{ord}_{q} \widetilde{Q}}\left(\sum_{a \in \mathbf{Z} / q^{e} \mathbf{Z}}(a \mid q)^{\operatorname{ord}_{q} \widetilde{Q}^{2 \pi i m a / q^{e}}}\right) \\
& =\prod_{q^{e} \| b}\left(\frac{-b}{q^{e}} \mid q\right)^{\operatorname{ord}_{q} \widetilde{Q}} \\
& \times\left(\sum_{c \in \mathbf{Z} / q \mathbf{Z}}(c \mid q)^{\operatorname{ord}_{q} \widetilde{Q}^{2 \pi i m c / q^{e}}} \sum_{d \in \mathbf{Z} / q^{e-1} \mathbf{Z}} \mathrm{e}^{2 \pi i m d / q^{e-1}}\right)
\end{aligned}
$$


where it is understood that in the above product $q$ is a prime dividing $b$. Unless $q^{e-1} \mid m$, the above sum over $d$ is zero; also, the sum over $c$ is -1 if $q^{e} \nmid m$ and $\operatorname{ord}_{q} \widetilde{Q}$ is even. Thus in the Fourier expansion of $E_{i}(\tau)$, the coefficient for $\mathrm{e}^{2 \pi i \tau}$ is

$$
\begin{aligned}
& \frac{(-1)^{k-1} 2 k \zeta(k)}{B_{k}} \\
& \sum_{\substack{n \in \mathbf{Z}_{+},(n, Q)=1 \\
n \text { square-free }}}(Q n)^{-k} H\left(\frac{1}{Q n}, L\right) \sum_{a \in(\mathbf{Z} / n \mathbf{Z})^{\times}}(-a \mid Q) \mathrm{e}^{2 \pi i a / b} \\
& =\frac{(-1)^{k-1} 2 k \zeta(k)}{Q^{k} B_{k}} H\left(\frac{1}{Q}, L\right) \prod_{q \mid Q}\left(-\frac{Q n}{q} \mid q\right)\left(\sum_{c \in \mathbf{Z} / q \mathbf{Z}}(c \mid q) \mathrm{e}^{2 \pi i c / q}\right) \\
& \left(\sum_{\substack{n \in \mathbf{Z}_{+},(n, Q)=1 \\
n \text { square-free }}} n^{-k} \chi_{L}(n)(n \mid Q)\right) .
\end{aligned}
$$

Note that $\left((-1)^{k-1} 2 k \zeta(k) / Q^{k} B_{k}\right) H\left(\frac{1}{Q}, L\right) \neq 0$. We know that

$$
\left|\sum_{c \in \mathbf{Z} / q \mathbf{Z}}(c \mid q) \mathrm{e}^{2 \pi i c / q}\right|=\sqrt{q}
$$

thus to show that the coefficient of $\mathrm{e}^{2 \pi i \tau}$ is nonzero, we only need to show that the last sum in the above expression is nonzero. We have

$$
\sum_{\substack{n \in \mathbf{Z}_{+},(n, Q)=1 \\ n \text { square-free }}} n^{-k} \chi_{L}(n)(n \mid Q)=1+\sum_{\substack{n>1 \\ n \text { square-free }}} n^{-k} \chi_{L}(n)(n \mid Q)
$$

and

$$
\left|\sum_{n>1} n^{-k} \chi_{L}(n)(n \mid Q)\right|<\sum_{n>1} n^{-2}<1
$$

5. Concluding remarks. Since the techniques used in this paper are in some ways rather general, one can imagine there are many extensions of the results presented here. First let us note that equation (82) of [5] (which we used in $\S 4$ to show $E_{i}(\tau) \neq 0$ in the case that $\mathbf{K}=\mathbf{Q}$, $\chi_{L}=1$ and $k>1$ ) is extended in [6] to allow $\mathbf{K} \neq \mathbf{Q}$; however, in [6] Siegel only concerns himself with free lattices. Thus the techniques of $\S 4$ can be used to show that the $E_{i}(\tau)$ are all nonzero in the case that 
the strict class number of $\mathbf{K}$ is 1 ; this author suspects that Siegel's results hold regardless of the strict class number of $\mathbf{K}$, and thus in general all the $E_{i}(\tau)$ are nonzero. Next, let us observe that Theorem 5.2.5 of [1] implies that the results of $\S 3$ can be extended to Siegel modular forms when $\mathbf{K}=\mathbf{Q}$ and $\chi_{L}=1$. In view of the techniques used to prove Theorem 7.4 of [7], this author suspects that Theorem 5.2 .5 of [1] can be extended to allow $\mathbf{K} \neq \mathbf{Q}$ and $\chi_{L} \neq 1$. If this is indeed the case, then the results of $\S 3$ herein may be extended to general Siegel modular forms. Furthermore, Siegel's papers [5] and [6] extend equation (82) of [5] to include Siegel modular forms attached to free lattices; thus the results of $\S 4$ could probably be extended to Siegel modular forms. Finally, we mention that these results should extend to include lattices of odd rank; this, in fact is the author's current concern.

\section{REFERENCES}

[1] A. N Andrianov, Quadratic Forms and Hecke Operators, Springer-Verlag, New York, 1987.

[2] M. Eichler, On theta functions of real algebraic number fields, Acta Arith., 33 (1977), 269-292.

[3] O. T. O'Meara, Introduction to Quadratic Forms, Springer-Verlag, New York, 1973.

[4] G. Shimura, The special values of the zeta functions associated with Hilbert modular forms, Duke Math. J., (1978), 637-649.

[5] C. L. Siegel, Über die analytische Theorie der quadratischen Formen, in "Gesammelte Abhandlungen", Springer-Verlag, New York, 1966, pp. 326-405.

[6] _ Über die analytische Theorie der quadratischen Formen III, in "Gesammelte Abhandlungen", Springer-Verlag, New York, 1966, pp. 469-548.

[7] L. H. Walling, Hecke operators on theta series attached to lattices of arbitrary rank, Acta Arith., 54 (1990), 213-240.

[8] On lifting Hecke eigenforms, Trans. Amer. Math. Soc. (to appear 1991).

Received January 22, 1990 and in revised form October 18, 1990.

UNIVERSITY OF COLORADO

BOULDER, CO 80309-0426 


\section{PACIFIC JOURNAL OF MATHEMATICS EDITORS}

\author{
V. S. VARADARAJAN \\ (Managing Editor) \\ University of California \\ Los Angeles, CA 90024-1555-05 \\ Herbert Clemens \\ University of Utah \\ Salt Lake City, UT 84112 \\ THOMAS ENRIGHT \\ University of California, San Diego \\ La Jolla, CA 92093
}

Nicholas ERCOLANI

University of Arizona

Tucson, AZ 85721

R. FINN

Stanford University

Stanford, CA 94305

VAUGHAN F. R. JONES

University of California

Berkeley, CA 94720

STEVEN KeRCKHOFF

Stanford University

Stanford, CA 94305
C. C. MOORE

University of California

Berkeley, CA 94720

Martin ScharlemanN

University of California

Santa Barbara, CA 93106

HAROLd STARK

University of California, San Diego

La Jolla, CA 92093

\section{ASSOCIATE EDITORS}
R. ARENS
E. F. BECKENBACH
B. H. NeUMANN
F. WolF
K. Yoshida (1906-1982)
(1904-1989)

SUPPORTING INSTITUTIONS

UNIVERSITY OF ARIZONA

UNIVERSITY OF BRITISH COLUMBIA

CALIFORNIA INSTITUTE OF TECHNOLOGY

UNIVERSITY OF CALIFORNIA

MONTANA STATE UNIVERSITY

UNIVERSITY OF NEVADA, RENO

NEW MEXICO STATE UNIVERSITY

OREGON STATE UNIVERSITY
UNIVERSITY OF OREGON

UNIVERSITY OF SOUTHERN CALIFORNIA

STANFORD UNIVERSITY

UNIVERSITY OF HAWAII

UNIVERSITY OF TOKYO

UNIVERSITY OF UTAH

WASHINGTON STATE UNIVERSITY

UNIVERSITY OF WASHINGTON 


\section{Pacific Journal of Mathematics}

\section{Vol. 151, No. $1 \quad$ November, 1991}

Fernanda Maria Botelho, Rotational entropy for annulus endomorphisms . . . 1

David F. Cowan, Some infinite chains in the lattice of varieties of inverse

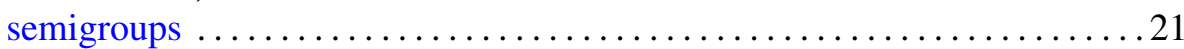

S. Greco and G. Raciti, The Lüroth semigroup of plane algebraic curves . . . 44

Kang-Tae Kim, Automorphism groups of certain domains in $\mathbf{C}^{n}$ with a

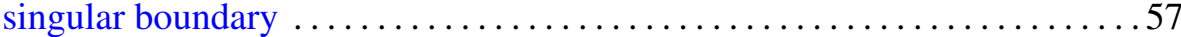

R. James Milgram, Surgery with finite fundamental group. I: The

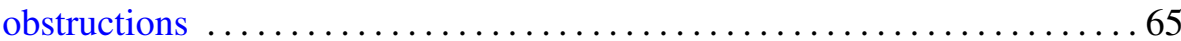

R. James Milgram, Surgery with finite fundamental group. II: The oozing

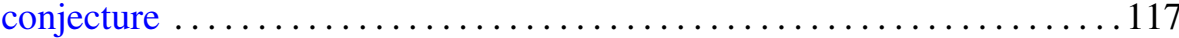

Shahriar Shahriari, On central type factor groups $\ldots \ldots \ldots \ldots \ldots \ldots \ldots 151$

Lynne Walling, Hecke eigenforms and representation numbers of quadratic forms ............................................... 179 